\title{
Automated Segmentation of the Corpus Callosum Midsagittal Surface Area*
}

\author{
${ }^{1}$ Flávio Luiz Seixas, ${ }^{2}$ Andrea Silveira de Souza, ${ }^{3}$ Alair Augusto Sarmet M. D. dos Santos, \\ ${ }^{1}$ Débora C. Muchaluat Saade \\ ${ }^{1}$ MidiaCom Lab, Telecommunications Engineering Department, \\ Universidade Federal Fluminense, Niterói, RJ \\ ${ }^{2}$ Radiology Division, LABS-Rede D'Or, Rio de Janeiro, RJ \\ ${ }^{3}$ Radiology Department, Faculdade de Medicina, UFF, Niterói, RJ \\ fseixas@midiacom.uff.br,andrea.silveiradesouza@gmail.com,alair@microlink.com.br, \\ debora@midiacom.uff.br
}

\begin{abstract}
The non-invasive in vivo nature of magnetic resonance imaging (MRI) makes it the modality of choice of many neuroanatomical imaging studies. This paper discusses automatic brain structure segmentation based on previous knowledge on statistical models. The method is validated by an experiment involving magnetic resonance images acquired from 20 healthy adult individuals (10 men and 10 women). The results provide normative data of the midsagittal surface area of the corpus callosum from a 46-55 years old range group, splitting results by gender. Our results were also compared with data obtained from other authors, validating the correlation between brain volume and the area of this structure. The final goal of this work is computer-aided diagnosis for brain diseases.
\end{abstract}

\section{Introduction}

The preference for non-invasive clinical diagnosis allied to computational improvements and high quality images obtained on magnetic resonance (MR) justify the increasing popularization of medical imaging exams in the neuroanatomy research area.

Some volumetric measurements acquired from magnetic resonance images are used to evaluate and quantify the impact of certain brain diseases on the human central nervous system [2][3]. Despite the acceptance of MR volumetric measurements as possibly reflecting ongoing brain diseases, this information is not routinely demanded by clinical or surgical specialists, as manual segmentation takes much time from the radiologist, besides being subjective and error-prone [4]. According to Marchetti et al [5], the time spent on manual segmentation of the hippocampus may take 75 minutes per patient/exam.

The goal of this work is to use image-processing methods and previous knowledge on statistical models to obtain automated segmentation and labeling of brain regions in order to support radiologists to make clinical diagnosis.

In this work, the automated segmentation was achieved by spatially normalizing all the structural images to the same stereotaxic space, segmenting the normalized images into gray matter, white matter and cerebral spinal fluid, and finally performing regionlabeling techniques. The normalization approach used voxel-based morphometry (VBM). The VBM technique allows us to compare different brains on a voxel-by-voxel basis after the deformation fields have been used to spatially normalize the images [1].

Our experiment analyzed 20 different MR scans (10 men and 10 women) obtained from OASIS database, with age ranging from 46 to 55 years old, in order to calculate the corpus callosum area. The corpus callosum is a prominent white matter bundle, readily identifiable on magnetic resonance imaging, which connects the two cerebral hemispheres in a homotopic organization with reference to the cortex representation [19]. Besides the corpus callosum key role in normative processes of inter-hemispheric communication and specialization [20], it is also important due to its vulnerability to environmental toxins, white matter diseases (such as multiple sclerosis) and schizophrenia [37, 21]. The size of the corpus callosum was also investigated by neurobiologists working in many specialized fields,

\footnotetext{
* This work has been partially supported by FAPERJ and CNPQ.
} 
including handedness [22], musical ability [23], schizophrenia [24], autism [25] and Alzheimer's disease [26][27].

We intended to verify gender differences of corpus callosum area obtained on a midsagittal plane. Similar studies were proposed by several different authors using MR manual segmentation or other dissection techniques $[6,7,8,9,10,11,12,13,14]$. Automatic segmentation of other specific brain regions, such as the hippocampus, may play an important role on Alzheimer's disease, even when symptoms are still subtle [4].

The rest of this article is organized in five more sections. Section 2 explains how automatic segmentation of brain tissue can be done using VBM techniques and anatomical atlas. Section 3 describes our practical experiments and presents the corpus callosum surface area of the 20 studied subjects, splitting results by gender and different age ranges. Section 4 evaluates our results. Section 5 compares our proposal to related work. Final considerations and future works are presented in Section 6.

\section{Brain Tissue Segmentation Based on Anatomical Atlas}

Segmentation using anatomical atlas [15] is based on previously knowledge of the image signal intensity and spatial probability distribution of the anatomical structures. This method became possible due to improvements in image normalization and morphometry techniques, as detailed in the following paragraphs.

Image normalization is the process of transforming different data sets into one coordinate system [16]. Normalization is necessary to compare or integrate data sets obtained from different measurements. The result of an image registration process is a set of matrices describing linear and non-linear transformations. Those linear transformations consist of a combination of 12 affine parameters representing translation, rotation, global scaling, shearing and perspective effects. The non-linear or non-rigid transformation approaches include polynomial wrapping and interpolation of smooth basis functions. The result is a deformation mapping built by linear combination of discrete cosine transforms. The optimal transformation parameters were achieved by applying a Gauss-Newton optimization method [18]. The goal is to transfer the original image to the same stereotaxic space of the reference image, called template [16].

The reference image represents the common brain shape considering different populations. The template was designed over voxel-based morphometry. VBM is a neuroimaging analysis technique that allows investigation of brain volume variability considering different individuals. The reference image is smoothed, so that each voxel represents the average of itself and its neighbors [15]. Voxel is a volume unit, representing a value on a regular grid in a tridimensional space.

Equation 1 represents the mathematical representation of image normalization, where $g_{\phi}$ is the spatial transfer function composed by parameter $\phi ; h_{\psi}$ is the intensity transfer function composed by parameters $\psi, I_{t}$ is the reference image and $\gamma$ is the translation matrix used to align source coordinates. The parameters $\phi$ are the 12 parameters of an affine transformation function, including parameters from a deformation field function. The parameters $\psi$ compose the intensity mapping function. The objective function consists of estimating parameters $\phi$ e $\psi$ in order to approximate the terms of equation 1.

$$
I=h_{\psi}\left(I_{t}\left(g_{\phi}(x, y, z)\right)\right)+\gamma
$$

Equation 2 is the problem formulation, where $B_{t}$ is the region of interest mapped at the template image and $\hat{\mathrm{B}}$ expresses the same region in the original image.

$$
\hat{B}(x, y, z)=B_{t}\left(g_{\phi}(x, y, z)\right)
$$

The normalization computational approach is achieved by a linear algorithm, whose function is to minimize the square-difference between the original image and the template, as showed in expression 3. Matrix $M$ represents the transformation parameters, $\mathrm{f}\left(\mathrm{Mx}_{\mathrm{i}}\right)$ is the original image, $\mathrm{g}\left(\mathrm{x}_{\mathrm{i}}\right)$ is the template and w, a constant.

$$
\sum_{i}\left[f\left(M x_{i}\right)-w g\left(x_{i}\right)\right]^{2}
$$

Image segmentation refers to the process of partitioning a digital image into multiple regions. In neuroanatomy, image segmentation classifies voxels into three tissue categories: gray matter, white matter and cerebral spinal fluid. The process uses a modified Gaussian model by knowing the prior spatial probability of each brain tissue categorization. Equation 4 expresses the probability of a voxel belonging to category $\mathrm{k}$, following a bayesian model [17], where $r_{i j k}$ is the probability function of the template and $\mathrm{s}_{\mathrm{ijk}}$ is the probability function of the original image [18]. The indices $\mathrm{i}$ and $\mathrm{k}$ represent the coordinates (abscissa and ordinate) of a slice from the normalized image.

$$
p_{i j k}=\frac{r_{i j k} s_{i j k}}{\sum_{l=1}^{K} r_{i j l} s_{i j l}}
$$

The segmentation computational approach is achieved by a linear algorithm, whose function is to maximize expression 5 . 


$$
\sum_{i=1}^{I} \sum_{j=1}^{J} \log \left(\sum_{k=1}^{K} r_{i j k} s_{i j k}\right)
$$

As the corpus callosum is a white matter structure, in the following step, we applied the automated segmentation based on the white matter tissue. This process is achieved by grouping regions after applying a region clustering technique. The major area region represents the corpus callosum. A cropping rectangular window around the corpus callosum was applied to delimit the regions considered. That window was necessary to eliminate the brainstem area from segmented image. The brainstem is another brain structure, whose area is bigger than the corpus callosum. The cropping window coordinates are the same for all images. Those coordinates were defined based on the template image, eliminating the need for user interaction in the segmentation process.

The final step is transferring back the corpus callosum region to the original image coordinate system by applying the inverse deformation mapping. The corpus callosum area is computed by counting its pixels and multiplying them by their spatial dimensions.

Figure 1 and Figure 2 show the normalization and segmentation processes, respectively. Figure 3 and Figure 4 show the corpus callosum (CC) region identification and labeling achieved by applying the inverse deformation mapping in order to transfer back the image to its original coordinate system.

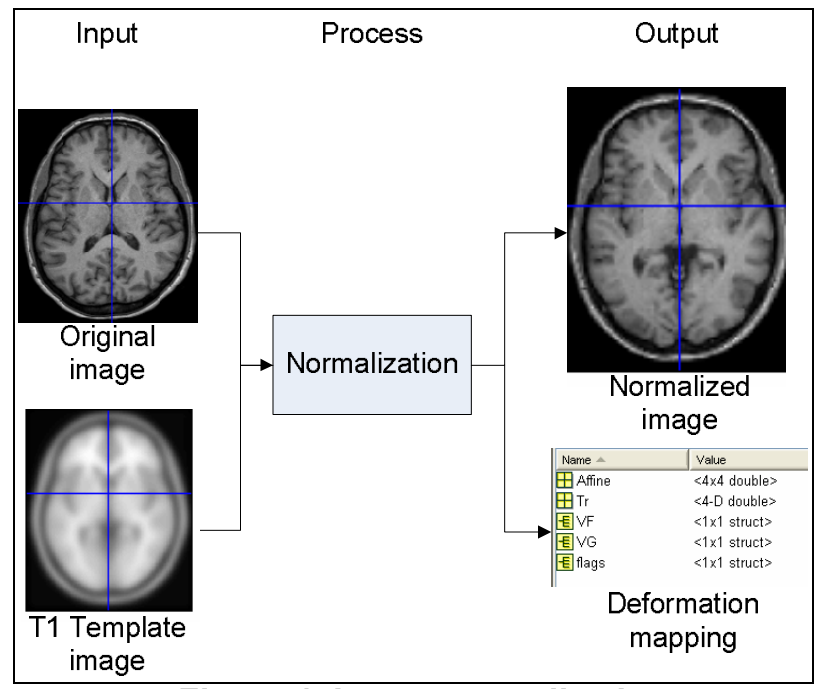

Figure 1. Image normalization

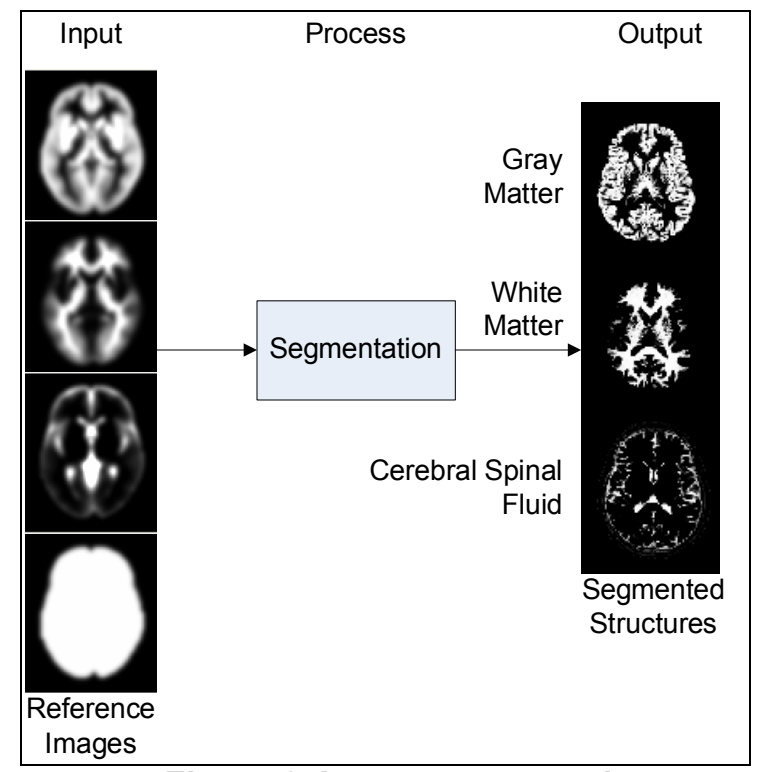

Figure 2. Image segmentation

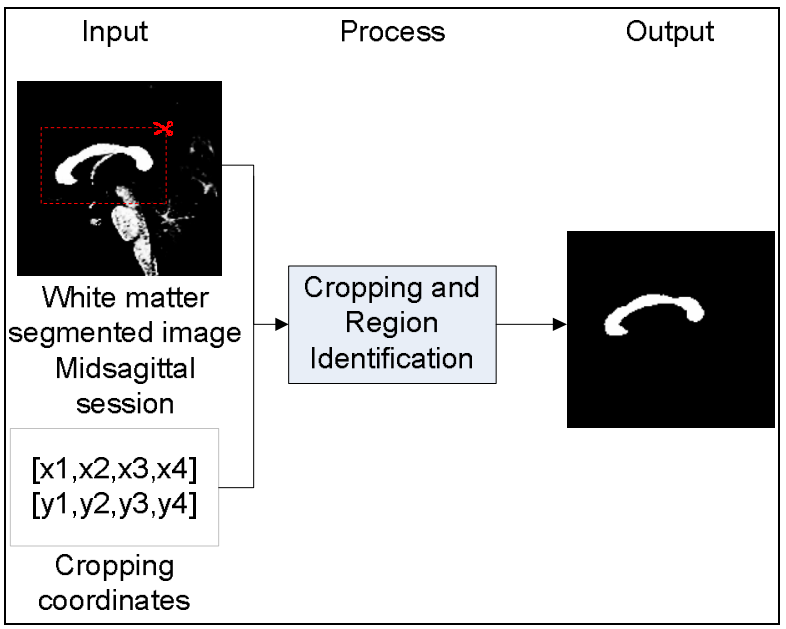

Figure 3. CC region identification

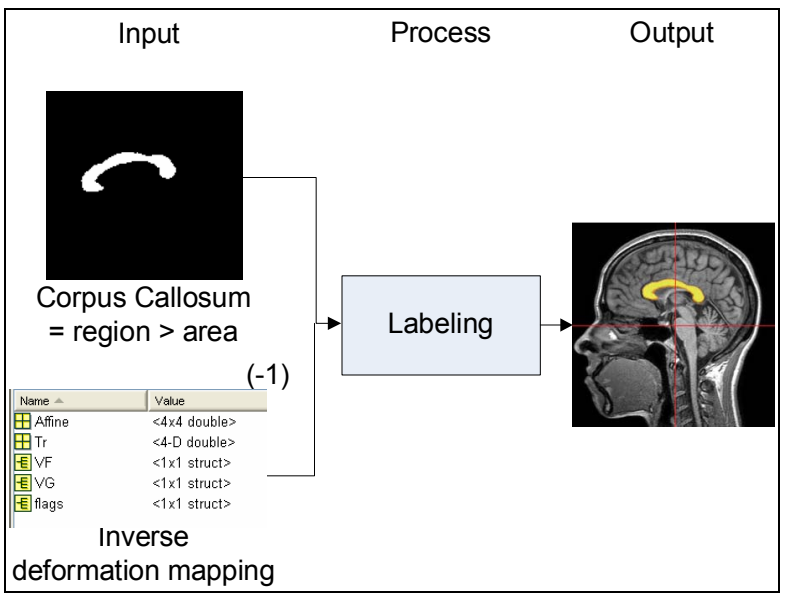

Figure 4. CC labeling 


\section{Corpus Callosum Segmentation Results}

The MR images used in this work were acquired from OASIS database, a public compiling and distributing MRI datasets facility, available by HHMI at Harvard University, NRG at Washington University School of Medicine and Biomedical Informatics Research Network, BIRN [28].

The analyzed images were from 20 non-demented right handed subjects (50\% males and 50\% females), with age ranging from 46 to 55 years old. The images format was 16-bit Analyze 7.5 with dimensions $256 \mathrm{x}$ $256 \times 128$, voxel size $1 \times 1 \times 1.25 \mathrm{~mm}$, oriented in the sagittal plane.

The VBM images were made available by MNI (Montreal Neurological Institute) as part of ICBM (International Consortium for Brain Mapping), NIH P20 project [29].

Our implementation utilized Mathworks Matlab and the SPM (Statistical Parametric Mapping) framework, developed by College London University [30].

The routines were processed on a Pentium $2.4 \mathrm{GHz}$, 1GB RAM, taking about 3 minutes to complete the normalization and segmentation processes for each image.

Figure 5 shows final corpus callosum segmentation results of 10 female subjects. Figure 6 shows results considering 10 male subjects. Finally, Table 1 shows the total brain volume (TBV), midsagittal corpus callosum (CCA) surface area and a ratio analysis between them (CCA/TBV), considering the entire image dataset. The ratio CCA/TBV was proposed to permit comparisons of different populations $[1,32]$.

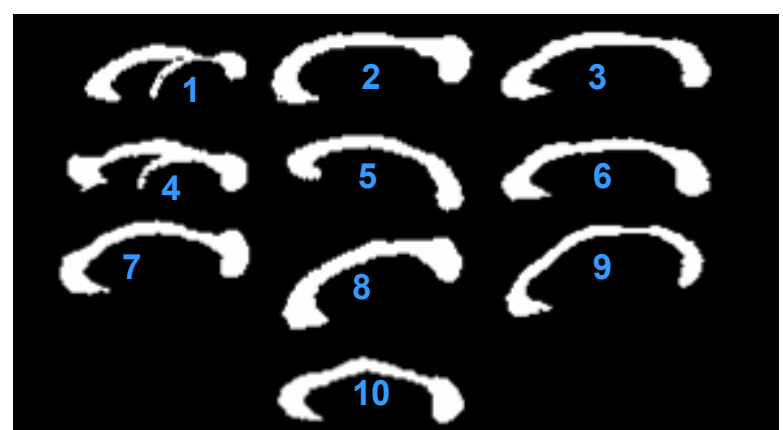

Figure 5. Corpus callosum segmented from female subjects

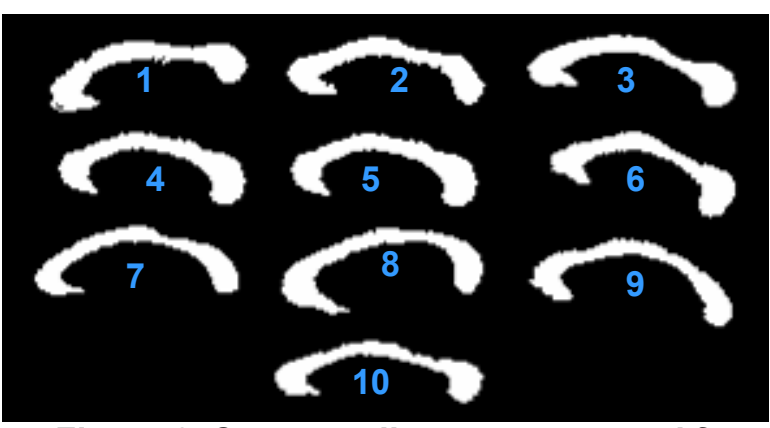

Figure 6. Corpus callosum segmented from male subjects

Table 1. Total brain volume, corpus callosum surface area and CCA/TBV ratio, splitting results by gender.

\begin{tabular}{|c|c|c|c|c|}
\hline & & $\begin{array}{c}\text { TBV } \\
\left(\mathrm{dm}^{3}\right)\end{array}$ & $\begin{array}{l}\text { CCA } \\
\left(\mathrm{cm}^{2}\right)\end{array}$ & $\begin{array}{c}\text { CCA / } \\
\text { TBV } \\
\text { ratio } \\
\left(10^{-3} \mathrm{~cm}^{-1}\right)\end{array}$ \\
\hline \multirow{11}{*}{ 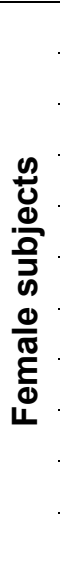 } & 1 & 1.27 & 4.42 & 3.48 \\
\hline & 2 & 1.38 & 6.62 & 4.80 \\
\hline & 3 & 1.35 & 5.98 & 4.42 \\
\hline & 4 & 1.37 & 5.59 & 4.06 \\
\hline & 5 & 1.39 & 5.13 & 3.70 \\
\hline & 6 & 1.56 & 6.18 & 3.97 \\
\hline & 7 & 1.46 & 6.05 & 4.13 \\
\hline & 8 & 1.35 & 6.69 & 4.97 \\
\hline & 9 & 1.42 & 5.18 & 3.65 \\
\hline & 10 & 1.41 & 5.46 & 3.87 \\
\hline & average & $1.39 \pm 0.076$ & $5.73 \pm 0.71$ & 4.10 \\
\hline \multirow{11}{*}{ 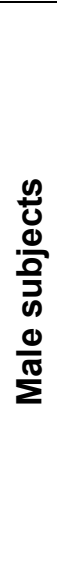 } & 1 & 1.60 & 7.01 & 4.37 \\
\hline & 2 & 1.71 & 6.65 & 3.88 \\
\hline & 3 & 1.63 & 7.17 & 4.41 \\
\hline & 4 & 1.58 & 7.06 & 4.46 \\
\hline & 5 & 1.52 & 6.51 & 4.28 \\
\hline & 6 & 1.69 & 5.84 & 3.46 \\
\hline & 7 & 1.59 & 6.14 & 3.87 \\
\hline & 8 & 1.76 & 7.34 & 4.16 \\
\hline & 9 & 1.57 & 6.48 & 4.12 \\
\hline & 10 & 1.61 & 6.62 & 4.09 \\
\hline & average & $1.67 \pm 0.082$ & $6.62 \pm 0.53$ & 4.09 \\
\hline \multicolumn{2}{|c|}{$\begin{array}{c}\text { final } \\
\text { average }\end{array}$} & $1.51 \pm 0.137$ & $6.17 \pm 0.76$ & 4.10 \\
\hline
\end{tabular}

$\mathrm{TBV}$ - total brain volume; CCA - corpus callosum area 


\section{Results Evaluation}

Sullivan et al [31] predicted that some differences in outcomes for corpus callosum area, considering different genders, would be significantly attenuated or entirely removed with adjustment based of brain sizes. These results can be observed in Table 1, comparing the CCA/TBV ratio between genders.

Salat et al [12] divided the corpus callosum in 3 sectors: anterior, middle and posterior sectors. They examined gender differences in area and age-related atrophy of the corpus callosum. The areas of the CC and $\mathrm{CC}$ sectors between genders were compared with and without head size correction. Despite differences in $\mathrm{CC}$ sectors, the adjusted $\mathrm{CC}$ areas were close, considering different genders.

Smith et al [32] argued that the corpus callosum midsagittal area depends on brain volume. Hence, it is necessary to consider certain ratios involving brain and corpus callosum, in order to allow comparisons of the corpus callosum size among groups or individuals who have different brain volumes.

Johnson et al [10] presented the corpus callosum area of 200 individuals based on MR manual segmentation, without head size correction. Table 2 shows its values, arranged by gender and age.

Table 2. CC manual segmentation based on 200 subjects according to Johnson et al. [10]

\begin{tabular}{cllll}
\hline Age range & \multicolumn{2}{l}{ Men } & \multicolumn{3}{c}{ Women } \\
\cline { 2 - 5 } & $\mathbf{N}$ & Area $\left[\mathbf{c m}^{2}\right]$ & $\mathbf{N}$ & Area $\left[\mathbf{c m}^{2}\right]$ \\
\hline $16-25$ & 30 & $6.80 \pm 0.96$ & 19 & $6.58 \pm 1.07$ \\
\hline $26-35$ & 20 & $6.94 \pm 1.02$ & 22 & $6.36 \pm 0.76$ \\
\hline $36-45$ & 14 & $6.78 \pm 1.16$ & 20 & $6.72 \pm 1.03$ \\
\hline $46-55$ & 18 & $6.76 \pm 1.08$ & 24 & $6.32 \pm 0.93$ \\
\hline $56-65$ & 18 & $6.16 \pm 0.83$ & 15 & $6.18 \pm 0.93$ \\
\hline $16-65$ & 100 & $6.70 \pm 1.02$ & 100 & $6.44 \pm 0.94$ \\
\hline CC- corpus callosum; - - number of individuals studied.
\end{tabular}

$\mathrm{CC}$ - corpus callosum; $\mathrm{N}$ - number of individuals studied.

Laissy et al [33] showed the corpus callosum area of 124 individuals without adjustment based on brain size, arranged by gender as condensed in Table 3 .

Table 3. CC manual segmentation based on 124 subjects according to Laissy et al [33]

\begin{tabular}{ccc}
\hline Gender & $\mathbf{N}$ & Area $\left[\mathbf{c m}^{2}\right]$ \\
\hline Men & 63 & $6.54 \pm 1.26$ \\
\hline Women & 61 & $6.17 \pm 1.07$ \\
\hline Total & 124 & $6.36 \pm 1.19$ \\
\hline CC - corpus callosum; $\mathrm{N}-$ number of individuals studied.
\end{tabular}

Evaluating those works from different authors, we concluded that our automated segmentation method results are similar to the literature. The CCA/TBV average ratios showed in Table 1 are very close, considering the corpus callosum area and total brain volume of individuals from different genders. The corpus callosum area itself is close to the values published by different authors that used manual segmentation. Manual segmentation depends on the technique used by the radiologists, leading to a few differences among areas obtained by different specialists. This may explain the difference among our area results and the ones showed in Table 2, especially for female images. On the other hand, our average CCA/TBV index values were very similar for both genders.

Evaluating Figure 5, besides the corpus callosum, another linear anatomical structure (the fornix) was identifiable in two analysed individuals (patients number 1 and 4). The fornix is a white matter structure located below to corpus callosum. In certain images, the signal intensity of these structures were very similar to the corpus callosum, so that the region identification algorithm incorrectly classified the fornix as belonging to corpus callosum. Despite being a small structure, its inclusion can be a method restrictor that can impact the final corpus callosum area analysis.

\section{Related Work}

Lundervold et al [34] proposed an automated segmentation of the corpus callosum in midsagittal sections, using both multispectral MRI measurements and prior information about shape (CC template). The algorithm has been successfully tested on a sample of 10 subjects scanned with multispectral MRI, collected from a study of dyslexia.

Hamarneh et al [35] presented an automatic segmentation model based on deformable organisms. It is a dynamic mesh model with physics-based framework that identifies object boundary considering its deformation parameters. The paper shows robust and consistent results.

Lee at al [36] proposed a new algorithm to find the corpus callosum automatically from midsagittal brain MR images using the statistical characteristics and shape information of the corpus callosum. A regiongrowing algorithm is applied in order to match previous shape information. The proposed algorithm was applied to 120 images and provided promising results.

The common characteristic of the works related above is the need of previous knowledge achieved by 
an initial shape or prototype in order to obtain the segmentation of the object of interest. This paper proposes a completely automated method to segment the corpus callosum, using a region identification technique from an MR midsagittal white matter segmented image. Hence, the knowledge of an initial shape or seed is not necessary in our method.

\section{Conclusions}

Volumetric measurements of brain structures can have several applications for diagnosis, tracking and treatment options of some diseases, which can demonstrate size and shape changes of certain anatomic structures, such as those observed in the corpus callosum and multiple sclerosis disease [37].

This work proposed the use of VBM techniques for automatic brain tissue segmentation and automatic calculation of the corpus callosum surface area from an MR midsagittal image. Our corpus callosum segmentation method is completely automatic. There is no need for a seed. We used a segmentation method based on anatomical atlas, which has a previously knowledge of the image signal intensity and spatial probability distribution of the anatomical structures.

The application of this work is useful to support future researches involving brain structures and their impact in the case of brain degenerative diseases. Another application can provide normative data in order to measure the impact of the corpus callosum atrophy and its damage to brain cognitive functions, correlating the anatomic volumes or areas with other signals or clinical tests.

In practice, the structure segmentation process is usually made manually by the medical specialist, which is time-consuming and error-prone. For this reason, automatic structure segmentation is certainly of great value for the medical staff.

The future challenge will be the design of a computer-aided diagnosis system that identifies brain structure anomalies and correlates them to specific brain diseases.

\section{References}

[1] Wright I. C., P. K. McGuire, J. B. Poline, et al. "A voxel-based method for statistical analysis of gray and whiter matter density applied to schizophrenia", Neuroimage, 1995, 2, pp. 244-252

[2] Tiffany Chaim M., Fábio L. S. Duran, Ricardo R. Uchida, Cíntia A.M. Périco, Cláudio C. de Castro, Geraldo, "Volumetric Reduction of the Corpus Callosum in Alzheimer's Disease in Invivo As Assessed with Voxel-
Based Morphometry", 2006, Psychiatry Research, Neuroimaging

[3] Bakshi R., V.S.R. Dandamudi, M. Neema, C. De, Robert A. Bermel, "Measurement of brain and spinal cord atrophy by magnetic resonance imaging as a tool to monitor multiple sclerosis", Journal of Neuroimaging, 2005, v15.4, pp. 30

[4] Bottino, Cássio Machado de Campos, Mário Rodrigues Louzã Neto, Cláudio Campi Castro, Regina Lúcia Elia Gomes, "Doença de Alzheimer, Transtorno Cognitivo Leve e Envelhecimento Normal: Avaliação por Medidas de Ressonância Magnética Volumétricas", Revista Psiquiatria Clínica, 2002, v.25, pp. 88-97

[5] Marchetti R. L. , Cássio Machado de Campos Bottino, Marie Nagahishi Suely, Cláudio Castro Campi, "Confiabilidade de Medidas Volumétricas de Estruturas Temporais Mesiais”, Arquivos de Neuro-Psiquiatria, 2002, v. 60, n.2B.

[6] Allen L. S., M. F. Richey, Y. M. Chai, R. A. Gorski, "Sex differences in the corpus callosum of the living human being“", J. Neuroscience, 1991, 11, pp. 933-942

[7] Byne W., R. Bleir, L. Houston, "Variations in human corpus callosum do not predict gender: a study using magnetic resonance imaging", Neurscience, 1988, 102, pp. $222-227$

[8] Constant D., H. Ruther, "Sexual dimorphism in the human corpus callosum? A comparison of methodologies", Brain Res., 1996, 727, pp. 99-106

[9] Jancke L., J. F. Staiger, G. Schlaug, Y. X. Huang, H. Steinmetz, „The relationship between corpus callosum size and forebrain volume“, Cerebral Cortex, 1997, 7, pp. 48-56

[10] Johnson S. C.,T. Farnworth, J. B. Pinkston, E. D. Bigler, D. D. Blatter, "Corpus callosum surface area across the human adult life span: effect of age and gender", Brain Research Bulletin, 1994, 35, pp. 373-377

[11] Kertsz A., M. Polk, S. E. Black, J. Howell, „Sex, handedness and morphometry of cerebral asymmetrics on magnetic resonance imaging“, Brain Res., 1990, 530, pp. 4048

[12] Salat D., A. Ward, J. A. Kaye, J. S. Janowsky, "Sex differences in the corpus callosum with aging", Neurobiol. Aging, 1997, 18, pp. 191-197

[13] Weis S., G. Weber, E. Wenger, M. Kimbacher, "The human corpus callosum and the controversy about a sexual dimorphism”, Psychobilogy, 1988, 16, pp. 411-415

[14] Weis S., G. Weber, M. Kimbacher, „The controversy about a sexual dimorphism of the human corpus callosum“, Int. J. Neuroscience, 1989, 47, pp. 169-173 
[15] Ashburner J., K. J. Friston, "Voxel-Based Morphometry", Neuroimage 11, 2000, pp. 805-821

[16] Friston K. J., J. Ashburner, C.D. Frith, J. B. Poline, J.D.Heather, R.S.J. Frackowiak, "Spatial Registration and Normalization of Images", Human Brain Mapping 2, 1995, pp. 165-189

[17] Ashburner J., K. J. Friston, "Multimodal Image Coregistration and Partitioning - a Unified Framework", NeuroImage 6, 1997, 209-217

[18] Ashburner J., "Computational Neuroanatomy", Thesis $\mathrm{PhD}$, University College London, 2000

[19] Pandya D. N., B. Seltzer, "The topography of commissural fibers", eds. Two Hemispheres-One Brain: Functions of the Corpus Callosum. New York: Alan R. Liss, Inc, 1986, pp. 47-74

[20] Trevarthen C., "Integrative functions of the cerebral commissures", eds. Handbook of Neuropsychology, 4. Amsterdam: Elsevier Scientific Publishers, 1990, pp. 49-83

[21] Barkhof F. J., M. Elton, J. Lindeboom, M. W. Tas, W. F. Schmidt, O. R. Hommes, C. H. Polman, A. Kok, J. Valk, "Functional correlates of callosal atrophy in relapsingremitting multiple sclerosis patients: a preliminary MRI study", J. Neurology, 1998, 245, pp. 153-158

[22] Habib M., D. Gayraud, A. Oliva, J. Regis, G. Salamon, R. Khalil, "Effects of handedness and sex on the morphology of the corpus callosum: a study with brain magnetic resonance imaging”, Brain and Cognition, 1991, 16, pp. 4161

[23] Lee D. J., Y. Chen, G. Schlaug, „Corpus callosum: musician and gender effects“, NeuroReport, 14, 2001, pp205-209

[24] Woodruff P. W. R., I. C. Manus, A. S. David, "Metaanalysis of corpus callosum size in schizophrenia", Journal of Neurology, Neurosurgery and Psychiatry, 1995, 58, pp. 457461

[25] Egaas B., E. Courchesne, O. Saitoh, "Reduced size of corpus callosum in autism", 1995, Archives of Neurology, 52, pp. 794-801

[26] Salat D., A. Ward, J. A. Kaye, J. S. Janawsky, "Sex differences in the corpus callosum with aging", Neurobiology, 1997, 18, pp. 191-197

[27] Biegon A., J. L. Eberling, B. C. Richardson, M. S. Rods, S. T. S. Wong, B. R. Reed, W. J. Jagust, "Human corpus callosum in aging and Alzheimer's diseaseÇ a magnetic resonance imaging study", Neurobiology of Aging, 1994, 4, pp. 393-397

[28] http://www.oasis-brains.org/
[29] International Consortium of Brain Mapping, http://www.loni.ucla.edu/ICBM

[30] Statistical Parametric Mapping Web Page, SPM, http://www.fil.ion.ucl.ac.uk/spm

[31] Sullivan E. V., Margaret J. Rosenbloom, John E. Desmond, Adolf Pfefferbaum, "Sex differences in corpus callosum size: relationship to age and intracranial size", Neurobiology of Aging, 2001, 22, pp. 601-611

[32] Smith R. J., F. Aboitiz, C. Schroter, R. Barton, V. H. Denenberg, R. Holly Fitch, B. L. Finlay, R. Holloway, W. L. Jungers, D. Wahlsten, K. M. Bishop, "Relative size versus controlling for size: interpretation of ratios in research on sexual dimorphism in the human corpus callosum", Anthropology, 2005, 46, 2, pp. 249-225

[33] Laissy J. P., B. Patrux, C. Dachateav, "Midsagital measurements of the corpus callosum in healthy subjects and diseased patients: a prospective study", Journal Neuroradiology, 1993, 14, pp. 145-154

[34] Lundervold A., Nicolae Duta, Torfinn Taxt, Anil K. Jain, "Model-Guided Segmentation of Corpos Callosum in MR Images", IEEE Computer Society Conference on Computer Vision and Pattern Recognition, CVPR, 1999, 1, pp. 1231

[35] Hamarneh T. McInerney. "Physics-Based Shape Deformations for Medical Image Analysis". SPIE-IS\&T Electronic Imaging: Image Processing: Algorithms and Systems, 2003, 5014, pp. 354-362

[36] Lee C., Terence A. Ketter, Michael Unser, "Automated Segmentation of Corpus Callosum in Midsagittal Brain Magnetic Resonance Images", Society of Photo-Optical Instrumental, 2000, 39, 4, pp. 924-935

[37] Simon J. H., L. D. Jabocs, M. K. Campion, R. A. Rudick, D. L. Cookfair, R. M. Herndon, J. R. Richert, A. M. Salazar, J. S. Fischer, D. E. Goodkin, N. Simonian, M. Lajaunie, D. E. Miller, K. Wende, A. Martens-Davidson, R. P. Kinkel, F. E. Munschauer, "A longitudinal study of brain atrophy in relapsing multiple sclerosis", American Academy of Neurology, 1999, 53, 1, pp. 139-148 